\title{
Does Robotic Knee Surgery Benefit a Patient's Recovery After TKA Surgery Compared with Conventional Methods?
}

\author{
Patrick Wilson $^{1}$, James Sires ${ }^{2}$ and Christopher Wilson ${ }^{3 *}$ \\ ${ }^{1}$ Research Student, Scotch College, South Australia \\ ${ }^{2}$ Orthopaedic Intern, Department of Orthopaedics, Flinders Medical Centre, South Australia and College of Medicine and \\ Public Health, Flinders University, South Australia \\ ${ }^{3}$ Consultant Orthopaedic Surgeon, Department of Orthopaedics, Flinders Private Hospital, South Australia and College of \\ Medicine and Public Health, Flinders University, South Australia
}

\begin{abstract}
Introduction: Total Knee Arthroplasty (TKA) is a very common procedure in the treatment of painful osteoarthritis of the knee. However, despite this, up to $20 \%$ of patients report dissatisfaction with the outcome of their procedure. Robotic TKA has been developed to improve the accuracy of the surgical procedure and improve intra-operative ligament balancing, which may improve patient function and satisfaction. The aim of this study was to evaluate if patients that underwent robotic TKA are more satisfied and have improved patient reported outcomes compared to those undergoing conventional TKA methods in the short-term.
\end{abstract}

Methods: Oxford knee score (OKS) and patient's satisfaction questionnaires were sent to 27 Patients who were approximately 6 months post robotic TKA surgery. The results from these surveys was compared with similar data obtained from a cohort of 20 age and sex matched patients who have had conventional non-robotic TKA surgery in the same hospital using the same implants. The data was then analysed to allow comparisons to be made regarding oxford knee scores and patient satisfaction between the 2 groups.

Results: Responses were obtained from 20 patients that underwent robotic TKA, which were compared with results from 20 patients that underwent conventional TKA. The average age in the robotic group was 73.6 with a range of 60 - 95 and the average age in the conventional group was 74.6 with a range of $56-90$. The mean 0KS was highest in the robotic group (32.3 vs 26.4). The difference in these values was statistically significant, $p$ value $=0.039$. A higher percentage of the robotic group had an oxford knee score $>30$, (70\% vs 40\%) with a $p$ value $=0.059$. When analysing pain, $40 \%$ of patients for robotic knee surgery reported no pain, compared with only $5 \%$ of patients in the conventional group. This result was also statistically significant with a $p$ value of $<0.01$.

Conclusion: Robotic knee surgery does benefit a patient's recovery postoperatively compared with traditional knee surgery. Overall, robotic arm assisted Total Knee Arthroplasty was associated with improved patient satisfaction, decreased pain and improved early functional recovery compared with Conventional Total Knee Arthroplasty.

KEYWORDS: Robotic knee surgery; Oxford knee score; Total knee arthroplasty; Patient reported outcomes

Quick Response Code:

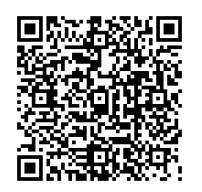

Address for correspondence: Christopher Wilson, Consultant Orthopaedic Surgeon, Department of Orthopaedics, Flinders Private Hospital, South Australia

Received: May 30, $2020 \quad$ Published: June 10, 2020

How to cite this article: Wilson P, Sires J and Wilson C. Does Robotic Knee Surgery Benefit a Patient's Recovery After TKA Surgery Compared with Conventional Methods?. 2020 - 2(3) OAJBS.ID.000180. DOI: 10.38125/OAJBS.000180 


\section{INTRODUCTION}

Total knee arthroplasty (TKA) is a very common surgical procedure, in 2018, over 56,000 of these procedures were performed in Australia. In 2018, the number of knee replacement cases has increased by $1.2 \%$ compared to 2017 . This surgical procedure is a significant expense for the healthcare budget and in 2018 , approximately $\$ 1.26$ billion was spent nationally on primary TKA procedures. However, not all patients are happy with the results of their knee surgery with recent studies suggesting up to $20 \%$ of patients are dissatisfied with the outcome of their surgery. Therefore, surgeons should be trying to improve techniques and the use of technology to reduce the number of patients that are dissatisfied with their outcomes.

Robotic surgery technology has evolved over recent years from Computer Assisted Surgical (CAS) Techniques. It is potentially more efficient and more accurate than conventional surgical methods [1-4]. Robotic surgery using the MAKO TKA uses a Computerised Tomography (CT) scan of the patient's knee before surgery to build a pre-operative plan of how the surgery should be performed. This determines where the robot will insert the new knee joint in the persons leg [5]. This scan involves multiple x-ray 'slices' of the patient's leg, which are then constructed into a 3D image using computer software. This data is then used by the MAKO software to build the pre-operative plan of where the knee implant should be inserted. An example of this pre-operative plan is shown in Figure1 and Figure 2 below. This allows the surgeon to visualise and adjust exactly where the implant will be inserted before the surgery has started. During the procedure, the surgeon finalises this plan by measuring several data points on the patient's actual knee, which is called bone registration, seen in Figure 3. This then allows a final plan to be made before any bone is removed. An example of this intra-operative plan is shown in Figure 4 below [5]. This plan specifically allows for the balancing of flexion and extension gaps between the bones. The scans can be evaluated before surgery and then it is fine-tuned intraoperatively once real-time bone mapping data has been added. This technology could allow surgeons to achieve a more accurate and reproducible bone resection, because of this, it leads to further accurate component and limb alignments $[4,6]$.

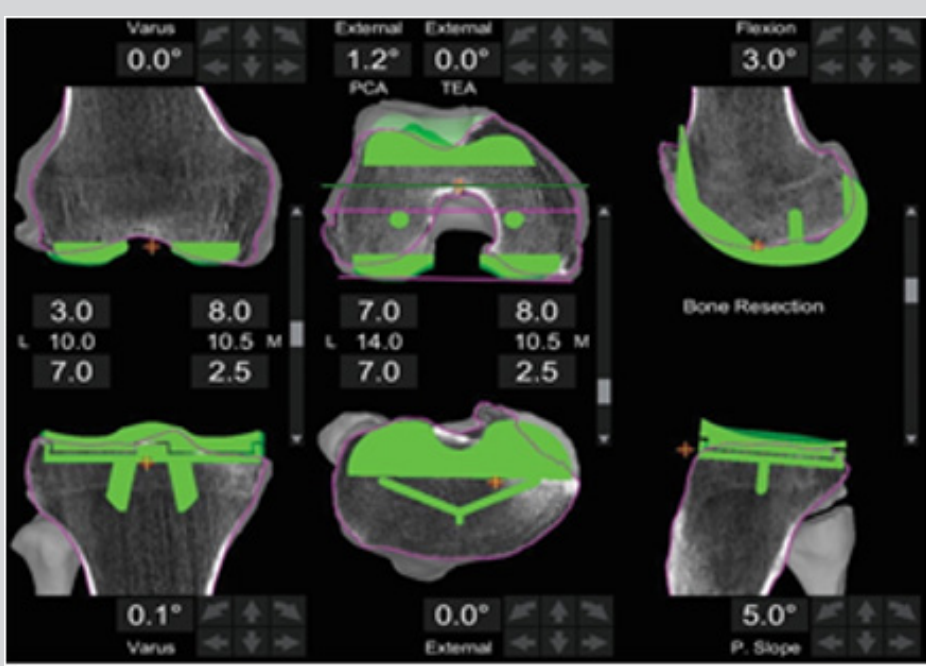

Figure 1: Preoperative plan showing planned bone resection of femur and tibia from computed tomography scan data.

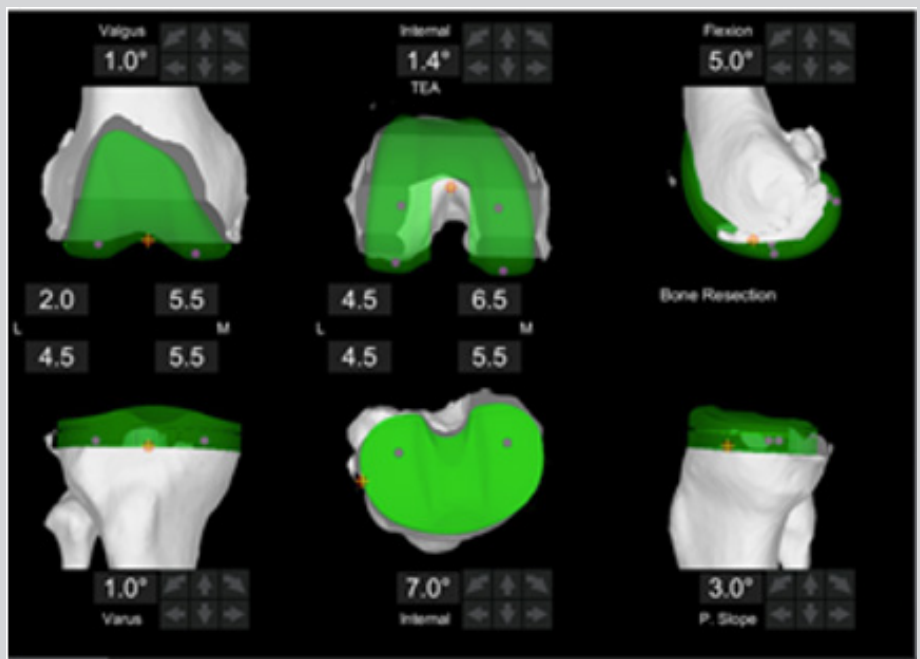

Figure 2: Preoperative plan showing expected position of implants prior to making bone resections from computed tomography scan data. 


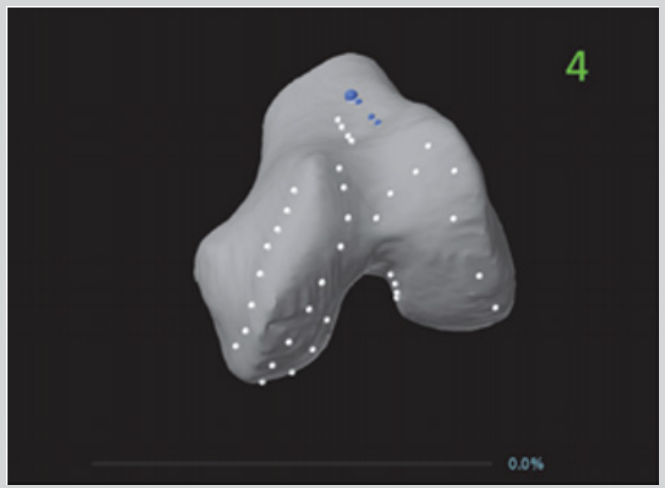

Figure 3: The mapping data points recorded on the patient's femur bone during surgery.

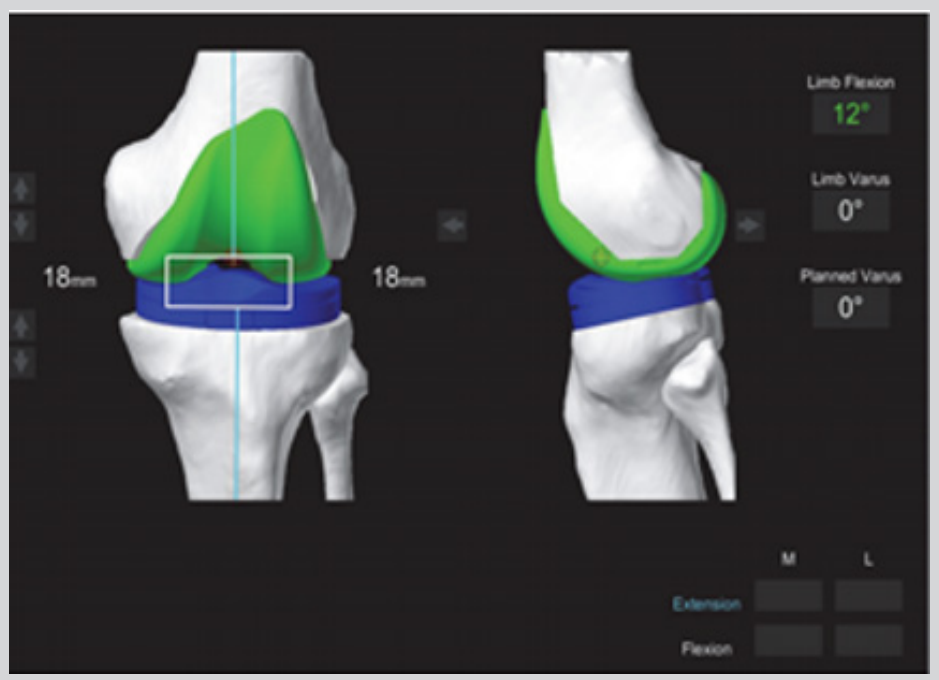

Figure 4: An example of the intra operative plan before the bony cuts are made.

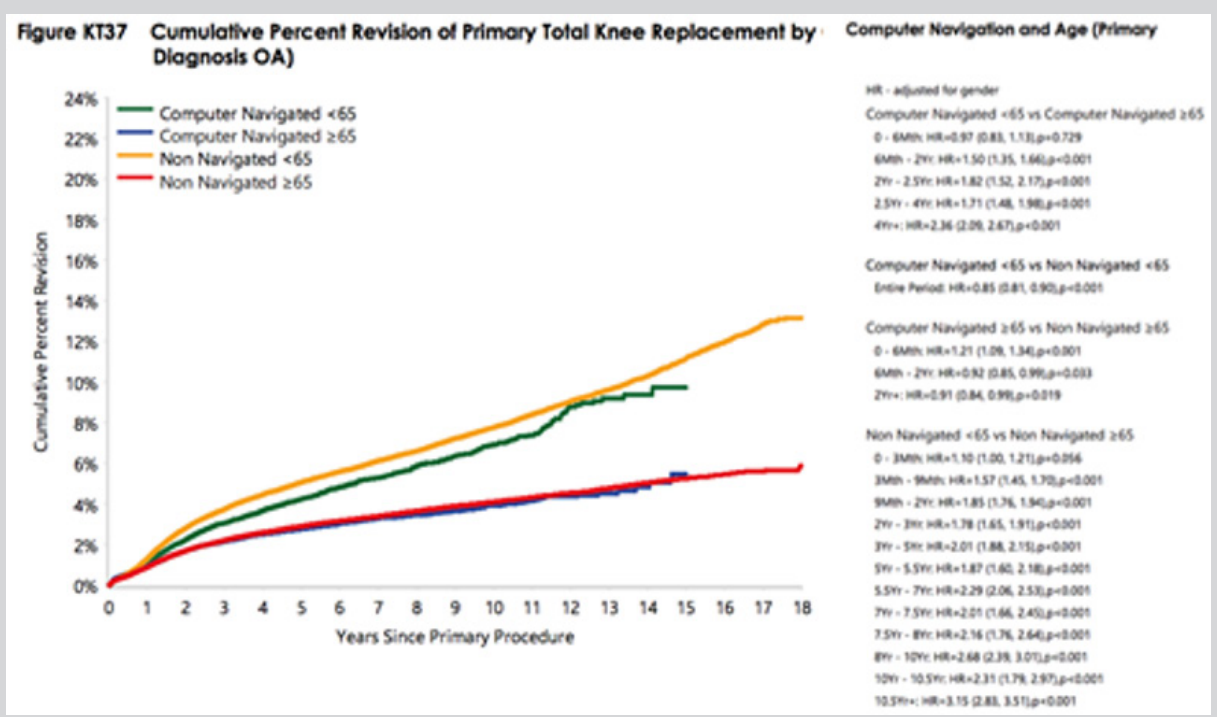

Figure 5: Improved survivorship in Computer Navigated TKA procedures in patients less than 65 years old P < 0.001 [7].

The bone cuts made by the MAKO Total Knee Robotic-Assisted Surgery are very accurate within fractions of a millimetre. The accuracy of both the femoral and tibial resections (cuts) are very high, with $94 \%$ of these cuts being within $\geq 1 \mathrm{~mm}$ of the original plan set before starting the procedure Sires et al. [4]. This level of precision suggests robotic knee surgery may be a technological advancement compared to traditional knee surgery. This procedure is not only very accurate, it delivers a new knee joint that is personalised by being designed on the patient's own knee anatomy. The surgeon is not only able to plan the exact position to implant 
the knee prosthesis but is also able to visualise how the knee will look, the patient's final alignment, the implants rotational profile and the balancing of the knee ligaments before performing the surgery. It is possible that this will allow patients to recover faster and have better function, but that is not proven at this time. Registry monitors all joint replacement procedures and provides useful data in the reasons why implants fail after surgery. Recent figures have shown improved survivorship in CAS Knee Arthroplasty compared to non-navigated techniques especially in younger patients less than 65 years old [7]. This is summarised in Figure 5. As robotic surgery is an evolution of the CAS technique, these surgeries may also benefit from improved survivorship, but long term follow up will be required to determine this outcome.

However, to assess patient function and satisfaction postoperative patient reported scores have been designed to evaluate improvements in pain, function and activities of daily living. These patients reported outcome measures (PROMs) are very useful in assessing satisfaction as they are patient centred and allow results to be collected in the short term and then compared to develop trends over time. The Oxford Knee Score (Appendix 1) is a validated 12-item patient reported PROMs survey, specifically designed and developed to assess the function and pain of patients after total knee replacement surgery. It is short, reproducible, and sensitive to clinically important changes. It can be used to evaluate patients function and satisfaction post operatively and allow comparison between different patients or groups of patients.A five point patient satisfaction questionnaire (Appendix 2.) was designed in our department to assess if patients are happy with their surgical outcome and if they would have the procedure again given the choice.

The aim of this study was to evaluate if patients who undergo Robotic TKA surgery perform better and are more satisfied compared to patients who have undergone surgery using conventional TKA methods in the short-term.

\section{METHODS}

This was a retrospective study of patients that have undergone
Robotic and Conventional TKA surgery. Prior to beginning data collection ethics approval was obtained from our local Ethics Committee, Ethics Approval Number: SCERP004. We then identified 27 Patients from our clinic records who have had robotic knee surgery and were approximately 6 months post-operative. All patients were sent a confidential consent form via email or hard copy and those that accepted this were asked to complete an Oxford Knee Score and a five point satisfaction questionnaire. Patients who did not initially respond were contacted one further time and invited to reply.

The data from these surveys was compared with similar data obtained from a cohort of 20 age and sex matched patients who have had conventional non-robotic TKA surgery in the same hospital using the same implants as part of a recent departmental audit. The data was then deidentified and summarised to allow comparisons to be made regarding the patient demographics, oxford scores and patient satisfaction between the 2 groups.

The results were collected and summarised using Microsoft Excel $^{\mathrm{TM}}$ which was also used to perform the calculations. Statistical analysis was performed using a "two-tail" T-Test or a Two-Sample test assuming unequal variances, to look for significant differences between the result of the two groups of patients. A $p$ value of $\leq 0.05$ was considered significant. This test was chosen as it is the most useful for comparing data in two different groups compared to, for example, two different sets of data on the same group of patients [8].

\section{RESULTS}

The 20 responses from those patients who had undergone robotic knee surgery and those who had undergone conventional knee surgery had similar demographics. For the robotic group the ratio of females to males was 11:9 and in the conventional group $3: 2$. The average age in the robotic-TKA group was 73.6 with a range of 60-95 and 74.6 with a range of 56-90 in the conventional group. Both groups of patients were therefore considered comparable with regards to age range and gender distribution. These results are summarised in Table 1 below.

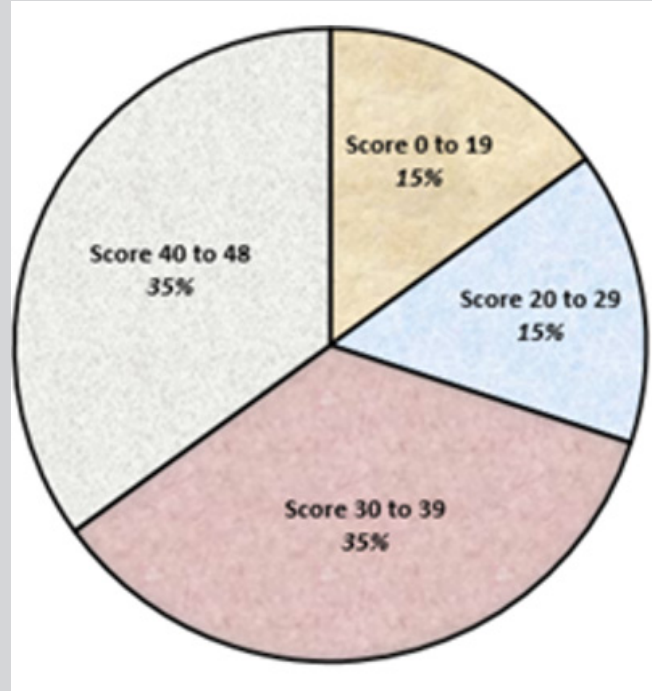

a

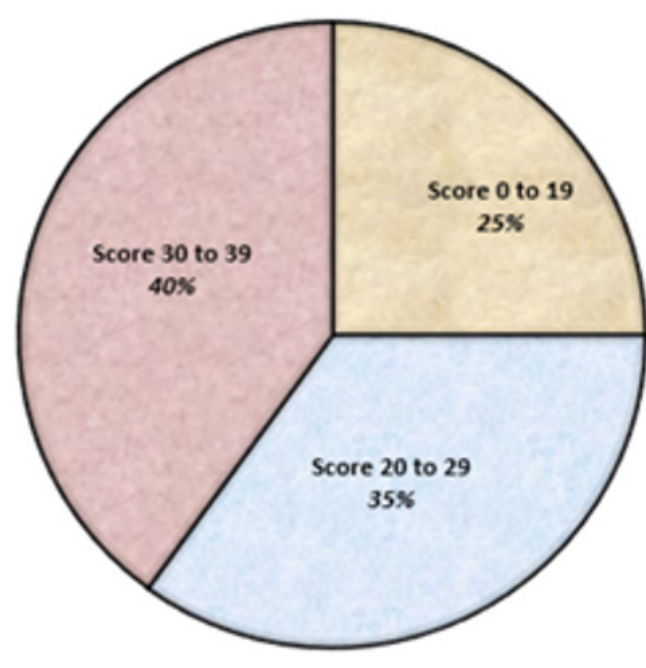

$\mathrm{b}$

Figure 6: a Oxford Knee Scores after robotic TKA expressed as a percentage.

b Oxford Knee Scores after conventional TKA expressed as a percentage 
Table 1: Patient Demographics.

\begin{tabular}{|c|c|c|c|c|c|}
\hline & Average Age & Age Range & No. Males & No. Females & Gender Ratio \\
\hline Robotic TKA & 73.6 & $60-95$ & 9 & 11 & $9: 11$ \\
\hline Conventional TKA & 74.6 & $56-90$ & 8 & 12 & $2: 03$ \\
\hline
\end{tabular}

The mean Oxford Knee Score was highest in the robotic group (32.3 vs 26.4). The difference in these values was statistically significant on a Two Tail t-test with a $\mathrm{p}$ value $=0.039$. A higher percentage of the robotic group had an Oxford Knee Score > $30(70 \%$ vs $40 \%)$ suggesting a good or excellent result [9]. Using a chi-squared test for statistical analysis this result was approaching statistical significance with a $p$ value $=0.059$. The distribution of oxford knee scores from both groups are summarised in Figure 6. These clearly show that most robotic knee surgery patient's scores are $>30$, whereas most conventional knee surgery patient's scores are $<30$.

When analysing a sub section of the oxford score results, "How would you describe the pain you usually have in your knee", we found 8 out of 20 patients for robotic knee surgery had no pain (40\%), compared to 1 out of 20 patients in the traditional knee surgery group (5\%). The difference between these two using a chisquared test groups was also statistically significant with a $p$ value of $<0.01$.

The final part of our questionnaire contained subjective questions in relation to patient satisfaction after their surgery from their own perspective. In this assessment, $70 \%$ of patients that underwent robotic total knee arthroplasty felt they were either satisfied or very satisfied with the outcome of their procedure. In addition, $60 \%$ of robotic patients stated they were very confident they would have this procedure again if they needed to do so.

\section{DISCUSSION}

When summarising the main points gained from this questionnaire-based study, the results are favourable showing that patients who underwent robotic assisted surgery had better postoperative recovery in the short-term. The Oxford Knee Score is based on activities of daily living suggesting that these patients had a greater improvement in their normal daily routine. Additionally, a higher percentage of patients in the robotic group reported no pain approximately 6 months post-operatively. Our survey was sent out to twenty-seven patients, and responses were received from twenty patients who had undergone robotic knee surgery. The survey used was the Oxford Knee Score and a set of 5 patient satisfaction questions. The aim of this study was to determine whether patients that underwent robotic TKA had improved recovery compared to Conventional TKA. Both groups of patients had similar demographics for age range and gender distribution as summarised in Table 1.

With regards to patient satisfaction, patients that underwent Robotic TKA were satisfied/very satisfied $70 \%$ of the time, with only $10 \%$ of patients being dissatisfied with their procedure. Previous publications have reported $20 \%$ dissatisfaction with their knee surgery in the long term, not just at 6 months [3]. These numbers are small due to the sample size but suggest overall, most patients are happy with the outcome of their procedure.

Patients usually describe satisfaction in a surgical clinic with regards to their ability to perform activities of daily living [3]. Pain is a common symptom after TKA surgery at 6 and even 12 months post op [10]. A comparison was made to see how many patients responded that they had no pain at all during their normal activities. In this sub-analysis, $40 \%$ of robotic knee surgery patients had no pain, whereas only $5 \%$ of conventional knee surgery patients had no pain. From a patient perspective having no pain is probably their most important outcome regarding their knee as this is predominant indication for the procedure. Therefore, the fact that a significantly higher proportion of patient's have no pain at all after their surgery, suggests these patients could mobilise quicker and return faster to their normal daily activities, work or hobbies.

The OKS is a validated tool for the assessment of a patient's function both before and after TKA surgery. It is also a patient reported outcome measure looking at the post-operative outcome from a patient's and not a surgeon's point of view [8]. The Oxford Knee Scores were higher in the robotic group compared to the conventional group, with the average Oxford Knee Score being significantly higher ( 32.3 vs 26.4 ). When comparing the proportions of each score, $70 \%$ of the robotic group exceeded 30 while only $40 \%$ of the conventional group achieved this score. A score of 30 or more is considered a good to excellent result [8]. This result failed to achieve statistical significance with a p value of 0.05 and it is unclear from this small sample size if this means there would be no difference or if this is a type 2 error that could be corrected with a larger sample in the future.

The higher scores obtained in the robotic group suggests these patients feel from their own perspective, that they perform better from a functional point of view in their normal routine. This suggests from this result we can show a significant improvement in that group about their post-operative function in relation to activities of daily living. These results combined, support the statement that the use of the robotic technique is not only an improvement in the technology, planning and accuracy of execution in TKA surgery, it delivers a knee that feels better, functions better and has less pain for the patient. The use of computer assisted surgery in the past has shown that TKA surgery can be performed with a higher level of accuracy, reduced numbers of surgical outliers with respect to component positioning and have significantly reduced failures in the long term $[10,11]$. If robotic surgery is seen as the next step in the development of computer assisted surgery hopefully it can deliver better results for our patients in the long term. However, long-term follow up with large numbers and the use of surgical registries will be required to confirm if this is in fact the case.

\section{CONCLUSION}

Robotic knee surgery does benefit a patient's recovery postoperatively compared with traditional knee surgery. Overall, robotic-arm assisted total knee arthroplasty was associated with improved patient satisfaction, decreased pain and improved early functional recovery compared with traditional jig-based surgery. 

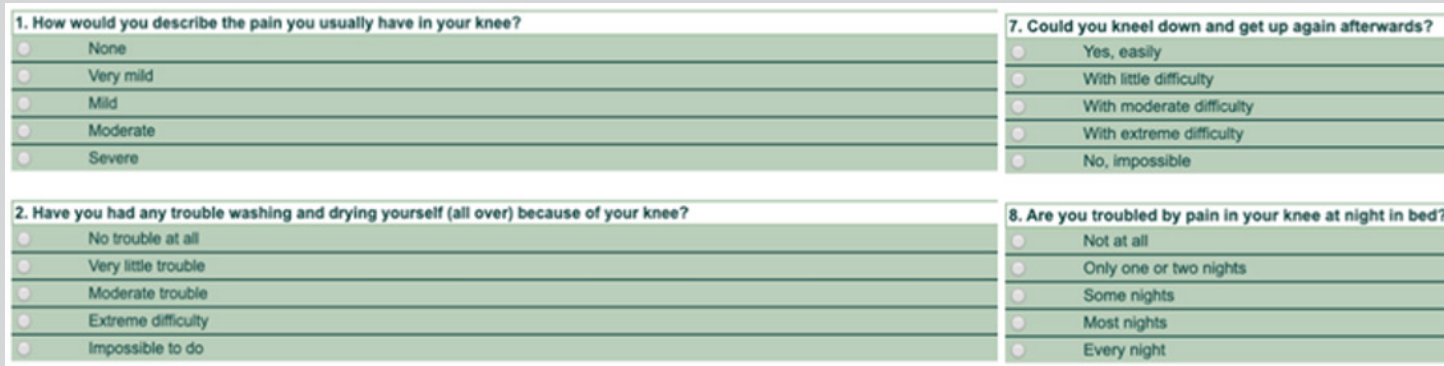

\begin{tabular}{l} 
3. Have you had any trouble getting in and out of the car or using public transport because of your knee? (With or without a stick) \\
No trouble at at \\
\hline Very little trouble \\
\hline Moderate trouble \\
\hline Extreme diflicully \\
\hline Impossible to do
\end{tabular}

\begin{tabular}{|} 
8. Are you troubled by pain in your knee at night in bed? \\
\hline Not at all \\
\hline Only one or two nights \\
\hline Some nights \\
\hline Most nights \\
\hline Every night \\
\hline
\end{tabular}

\begin{tabular}{|c|c|}
\hline & W long are you able to walk betore the pain in your knee becomes s eve re? (With or without a stick) \\
\hline 0 & No pain $>60 \mathrm{~min}$ \\
\hline o & 16.60 minutes \\
\hline o & 5.15 minutes \\
\hline 요 & Around the house only \\
\hline o & Not at all - severe on walking \\
\hline
\end{tabular}

\begin{tabular}{l} 
9. How much has pain from your knee interfered with your usual work? (including housework) \\
\hline Not at all \\
\hline A little bit \\
\hline Moderately \\
\hline Greatly \\
\hline Totally \\
\hline 0
\end{tabular}

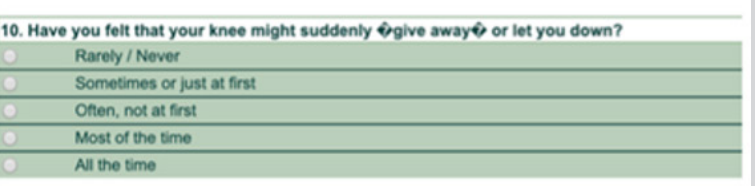

\begin{tabular}{l} 
5. After a meal (sat at a table), how painful has it been for you to stand up from a chair because of your knee? \\
\begin{tabular}{|l|}
\hline Not at all paintul \\
\hline Slightly painful \\
\hline Moderately pain \\
\hline Very paintul \\
\hline Unbearable
\end{tabular} \\
\hline
\end{tabular}

\begin{tabular}{|l|}
\hline 11. Could you do household shopping on your own? \\
\hline Yes, easily \\
\hline With ittle difficulty \\
\hline Wath moderate diffoulty \\
\hline Wath extreme difficulty \\
\hline No, impossible \\
\hline
\end{tabular}

\begin{tabular}{l} 
6. Have you been limping when walking, because of your knee? \\
Rarely / never \\
\hline Sometimes or just at frst \\
\hline Often, not just at first \\
\hline Most of the time \\
\hline All of the time \\
\hline
\end{tabular}

\begin{tabular}{|l|}
\hline 12. Could you walk down a flight of stairs? \\
Yes, easily \\
\hline Wath ittle difficulty \\
\hline Wath moderate difficulty \\
\hline Wath extreme difficulty \\
\hline No, impossible \\
\hline
\end{tabular}

Appendix 1: Oxford knee score with 12 questions related to patient function.

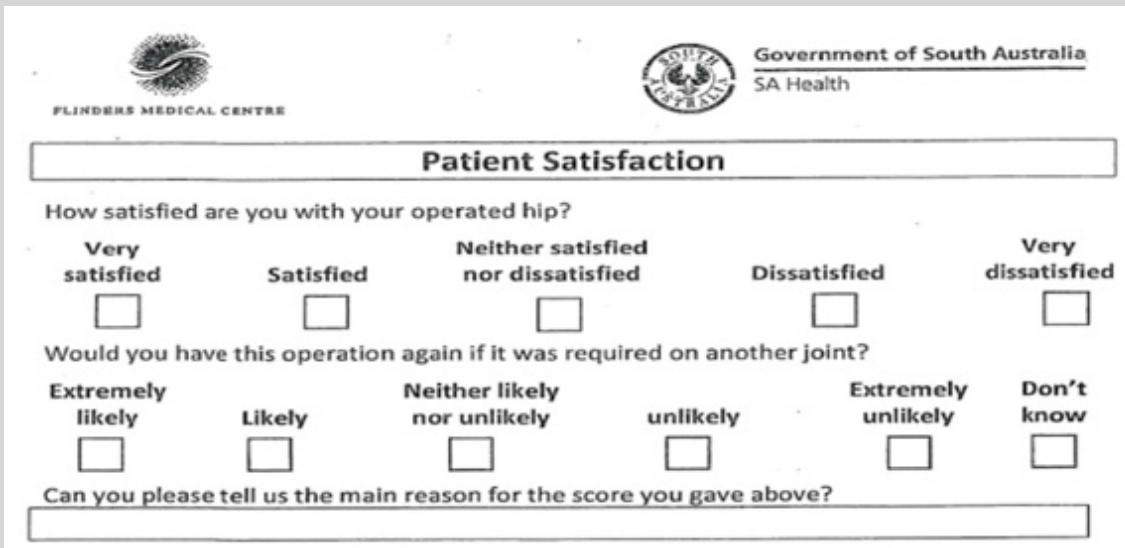

How likely are you to recommend our department to friends and family if they need similar care or treatment?

$\begin{gathered}\text { Extremely } \\ \text { likely }\end{gathered}$
$\square$

Can you please tell us why you would/would not recommend us to your friends and family?

EXPERIENCE SCORE: Out of 100 how would you rate your hospital experience with 0 being the "Worst" and 100 being the 'Best' possible.

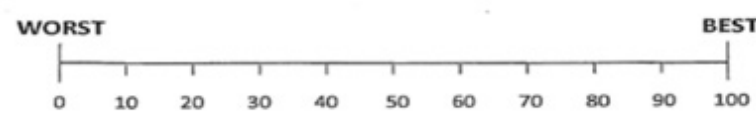

Please can you tell us the main reason for the score you have given?

Appendix 2: Local patient five-point Satisfaction questionnaire. 


\section{REFERENCES}

1. (2019) Australian national joint replacement registry, AOANJRR. Annual Report, 209.

2. Lavernia C, Lee DJ, Hernandez VH (2006) The increasing financial burden of knee revision surgery in the United States. Clin Orthop Relat Res 446: 221-226.

3. Gunaratne R, Pratt DN, Banda J, Fick DP, Khan RJK, et al. (2017) Patient dissatisfaction following total knee arthroplasty: A systematic review of the literature. J Arthroplasty 32(12): 3854-3860.

4. Wilson CJ, Sires JD, Craik JD (2019) Accuracy of bone resection in MAKO total knee robotic-assisted surgery. J Knee Surg.

5. Stryker (2016) Mako TKA surgical guide.

6. Wilson CJ, Sires JD (2020) CT Validation of intraoperative implant position and knee alignment as determined by the MAKO total knee arthroplasty System. J Knee Surg.
7. Dawson J, Fitzpatrick R, Murray D, Carr A (1998) Questionnaire on the perceptions of patients about total knee replacement. J Bone Joint Surg Br 80(1): 63-69.

8. (2020) Statistics for the rest of us, T test in excel easy steps with video.

9. Judge A, Arden NK, Kiran A, Price A, Javaid MK, et al. (2012) Interpretation of patient-reported outcomes for hip and knee replacement surgery. BJJ $94(3)$

10. Rice DA, Kluger MT, McNair PJ, Lewis GN, Somogyi AA, et al. (2018) Persistent postoperative pain after total knee arthroplasty: A prospective cohort study of potential Risk Factors. Br J Anaesth 121(4): 804-812.

11. Chauhan SK, Clark GW, Lloyd S, Scott RG, Breidahl W, et al. (2004) Computer-assisted total knee replacement. A controlled cadaver study using a multi-parameter quantitative CT assessment of alignment. J Bone Joint Surg Br 86(6): 818-823. 